\title{
Disseminated cryptococcosis with concomitant thoraco-abdominal involvement: a rare case report from India
}

\author{
Sushma Krishna ${ }^{1 *}$, Seemanthini Desai ${ }^{2}$, Paranthaaman ${ }^{2}$ and Shrivalli ${ }^{3}$ \\ *Correspondence: chummu.dummu@gmail.com \\ 'Department of Microbiology, Amrita Institute of Medical Sciences, Cochin-682041, India. \\ 2Department of Microbiology, Apollo Hospitals, Bangalore, India. \\ ${ }^{3}$ Department of Radiology, Apollo Hospitals, Bangalore, India.
}

\begin{abstract}
Infections by Cryptococcus neoformans usually involves the central nervous system and the respiratory tract. We report a case of disseminated cryptococcosis with cryptococcal empyema and liver abscess. The initial diagnosis was only ascites with hepatitis and or cholangitis of unknown cause. Routine chest X-ray film showed pleural effusion, and his retropositive status was detected for the first time. Dissemination was considered when the computerized abdomen scans carried out revealed multiple cystic lesion of the liver with an abscess with diffuse peritoneal thickening. Culture of pleural fluid and liver abscess aspirate grew C. neoformans. Patient improved symptomatically with antifungal therapy. This case illustrates that disseminated cryptococcosis has to be included in the differential diagnosis in a systemic infection with thoraco-abdominal involvement, more so in an immunocompromised patient. Early diagnosis with relevant investigations like computerized tomography scans plays a key role in the administration of anti-fungal therapy which can be life saving.
\end{abstract}

Keywords: Disseminated cryptococcosis, empyema, liver aspirate, india

\section{Introduction}

Disseminated cryptococcosis is known to occur in immunocompromised patients [1]. Lungs, nervous system and skin are the commonly involved organs and have been documented. Ascites with Pleural effusion are relatively rare manifestations of disseminated cryptococcosis [2-4]. We report one such case in HIV positive patient who responded to antifungal therapy for a while. To our knowledge, this comes as one of the rare reports describing the disseminated cryptococcosis concomitantly involving the thorax and abdomen.

\section{Case history}

A 35 year old man, non-alcoholic with no previously documented pre-morbid illness came with complaints of diffuse abdominal pain with post prandial nausea from ten days and yellowish discoloration of urine since one day. On examination, he was icteric, had bilateral pedal odema. Systemic examination revealed decreased air entry in the right lower chest zones and gross ascites with tenderness per abdomen. He was started on intravenous ciprofloxacin $200 \mathrm{mg} \mathrm{BD}$ and metronidazole 500 mg TDS. Investigations revealed deranged liver function tests, normal hemogram and serum creatinine. Hepatitis A IgM and serological markers for Hepatitis-B were negative. Routine HIV testing by ELISA showed positive, was confirmed with Western blot, followed by CD4 lymphocyte count which was $10 \mathrm{c} / \mathrm{mm}$. Chest $\mathrm{x}$-ray revealed blunting of right costophrenic angle suggesting pleural effusion which was immediately drained. Fluid was exudative, hemorrhagic, predominant lymphocytic with elevated lactate dehydrogenase levels (Figure 1). No acid fast bacilli were found on continuous smear microscopy. He was emperically started on modified anti-tuberculous treatment
(ATT) with ethambutol 200mgOD and ofloxacin $200 \mathrm{mg} \mathrm{BD}$. The ultrasound abdomen clearly showed hepatomegaly with multiple cysts in liver with thick wall of internal echoes, a larger cyst at the porta, compressing the ports with intrahepatic billary duct. Ascites was also noted. Ultrasound guided liver aspiration was done and sample was received for microbiological processing. $\mathrm{CT}$ whole abdomen plain and contrast carried out revealed multiple hyperdense lesion in both lobes of liver suggestive of hemorrhagic cysts, compression of portal vein and its branches, extensive subhepatic, omental thickening, stranding with irregular subhepatic collections as a sequelae of a ruptured hepatic cyst with mild ascites. Urine and blood culture remained sterile throughout. After 72 hours of incubation, culture of pleural fluid and the liver abscess aspirate grew spherical, budding mucoid yeast colonies which stained well with India ink preparation. Cryptococcus neoformans grown was sensitive to Amphotericin B, Fluconazole, Itraconazole, Voriconazole with intermediate sensitivity to Flucytosine, confirmed by API. Amphotericin B 50mg IV OD was started on day 6 of admission and he was managed conservatively. ATT was stopped. Patient seemed to be making an improvement symptomatically over the next few days. Antiretroviral therapy was planned for a later date. He opted to get discharged against the medical advice and did not return for further follow ups.

\section{Discussion}

The occurance and extent of pulmonary cryptococcosis in India is not well understood. Available literature on pulmonary cases has quoted underlying disease or immunodeficiencypatients with lymphoma, sarcoidosis, diabetes mellitus, those requiring immunosuppressive agents, those on corticosteroids 


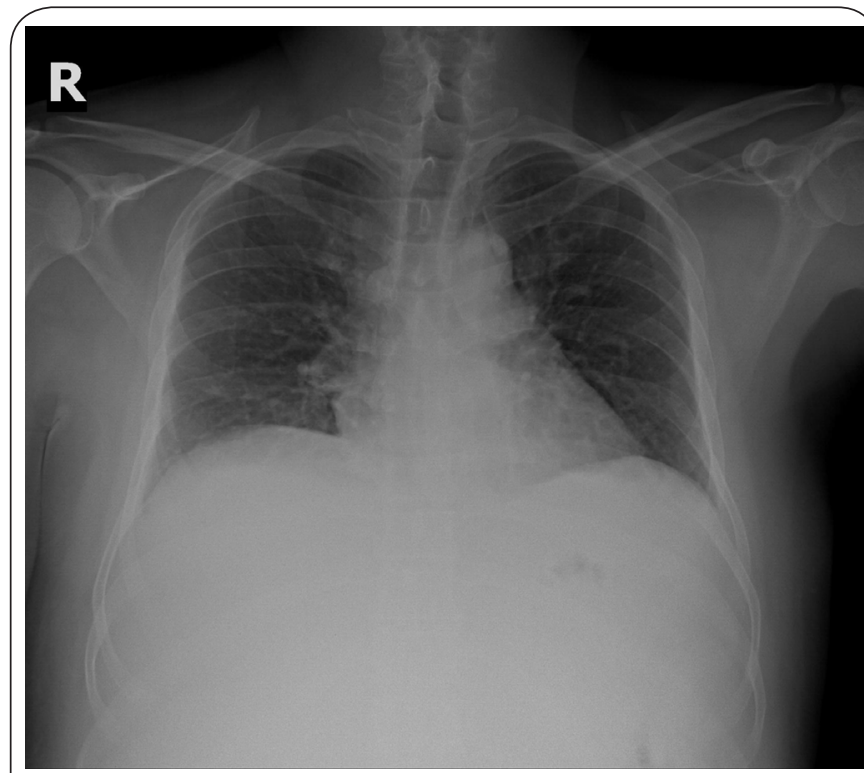

Figure 1. Chest X-ray of the patient showing right pleural effusion.

as pre-disposed to disseminated cryptococcemia. Largely asymptomatic like this patient, chest pain, shortness of breath and headache are also reported in immunocompromised patients of pulmonary cryptococcosis [5]. There is paucity of information concerning the natural history of pulmonary disease as well. Pleural effusion and parenchymal disease may co-exist in patients with progressive primary cryptococcal pulmonary disease. Effusion alone may be the presenting manifestation of cryptococcosis and may resolve without recurrence if the patient's immune response is appropriate [6]. A combination of Amphotericin B with flucytosine is recommended for the treatment of disseminated cryptococcosis [7] but no specific guidelines exist for empyema associated with cryptococal disease. Recovery was noted in this patient with Amphotericin B monotherapy along with aspirations and chest tube drainage. As the symptoms initially were not pathognomonic, the radiological findings prompted the treating clinicians to seek for a wider spectrum of causes to in a HIV positive patient and the diagnosis of pulmonary cryptococcosis was confirmed with the demonstration and isolation of C.neoformans in respiratory secretions. Also, there are increasing numbers of case reports augmenting the association of liver disease and development of cryptococcal peritonitis but this patient was not found to have any underlying liver disease and liver was involved as a part of dissemination $[\mathbf{8 , 9}]$. Detection of fungal antigens in pleural effusion, serum and demonstration of tissue invasion could have been useful in documenting the infection but was not done. The CT finding of low-attenuation lesions in the liver or spleen of patients with suspected systemic infection is not characteristic for fungal infection and may also be observed in patients with disseminated mycobacterial infection also
[10] and only a prompt microbiological contribution can help arrive at the correct diagnosis. It takes over 48-72 hours for Cryptococcus to grow on culture plates and only an alerted microbiologist with high index of suspicion will further incubate the plates, which are routinely discarded by 48 hours. Isolation of C.neoformans from any sample like respiratory or CSF in an immunocompromised host who presents with clinical picture of hepatitis or cholangitis of unexplainable cause must include cryptococcal liver infection in the list of differential diagnosis. Survival rate can potentially be improved with early diagnosis and antifungal therapy.

In conclusion, disseminated cryptococcosis should be included in the differential diagnosis of any patient with suspected systemic infection having thoraco-abdominal involvement more so in an immunocompromised patient. We would also like to conclude that a high index of suspicion with the relevant and timely investigations like CT and ultrasound scans help to administer early antifungal therapy in disseminated Cryptococcosis with multi-organ involvement.

\section{List of abbreviations \\ $\mathrm{CT}$ : computerized tomography}

\section{Competing interests}

The authors declare that they have no competing interests.

\section{Acknowledgement}

We sincerely thank Consultant Gastroenterologist and management, Apollo Hospitals, Bangalore and Dr. Rani Mary Varghese, AIMS, Cochin, India.

\section{Publication history}

Received: 10-Jan-2013 Revised: 28-Jan-2013

Accepted: 6-Mar-2013 Published: 19-Mar-2013

\section{References}

1. Kerkering TM, Duma RJ and Shadomy S: The evolution of pulmonary cryptococcosis: clinical implications from a study of $\mathbf{4 1}$ patients with and without compromising host factors. Ann Intern Med 1981, 94:6116. | Article | PubMed

2. Young EJ, Hirsh DD, Fainstein V and Williams TW: Pleural effusions due to Cryptococcus neoformans: a review of the literature and report of two cases with cryptococcal antigen determinations. Am Rev Respir Dis 1980, 121:743-7. | Article | PubMed

3. Katz AS, Niesenbaum $L$ and Mass B: Pleural effusion as the initial manifestation of disseminated cryptococcosis in acquired immune deficiency syndrome. Diagnosis by pleural biopsy. Chest 1989, 96:4401. | Article | PubMed

4. Poblete RB and Kirby BD: Cryptococcal peritonitis. Report of a case and review of the literature. Am J Med 1987, 82:665-7. | Article I PubMed

5. Chang WC, Tzao C, Hsu HH, Lee SC, Huang KL, Tung HJ and Chen CY: Pulmonary cryptococcosis: comparison of clinical and radiographic characteristics in immunocompetent and immunocompromised patients. Chest 2006, 129:333-40. | Article | PubMed

6. Kim DY, Kim Y, Baek SY and Yoon HK: Simultaneous thoracic and abdominal presentation of disseminated cryptococcosis in two patients without HIV infection. AJR Am J Roentgenol 2003, 181:1055-7. | Article | PubMed

7. Saag MS, Graybill RJ, Larsen RA, Pappas PG, Perfect JR, Powderly WG, Sobel JD and Dismukes WE: Practice guidelines for the management of cryptococcal disease. Infectious Diseases Society of America. Clin Infect Dis 2000, 30:710-8. | Article | PubMed 
Krishna et al. Microbiology Discovery 2013,

http://www.hoajonline.com/journals/pdf/2052-6180-1-3.pdf

8. Albert-Braun S, Venema F, Bausch J, Hunfeld KP and Schafer V: Cryptococcus neoformans peritonitis in a patient with alcoholic cirrhosis: case report and review of the literature. Infection 2005, 33:282-8. | Article | PubMed

9. Mabee $\mathrm{CL}$, Mabee SW, Kirkpatrick RB and Koletar SL: Cirrhosis: a risk factor for cryptococcal peritonitis. Am J Gastroenterol 1995, 90:2042-5. | Article | PubMed

10. Chakrabarti S, Varma S, Kochhar R, Gupta S, Gupta SK and Rajwanshi A: Hepatosplenic tuberculosis: a cause of persistent fever during recovery from prolonged neutropenia. Int J Tuberc Lung Dis 1998, 2:575-9. | Article | PubMed

\section{Citation:}

Krishna S, Desai S, Rg P and N S: Disseminated cryptococcosis with concomitant thoraco-abdominal involvement: a rare case report from India.

Microbiology Discovery 2013, 1:3.

http://dx.doi.org/10.7243/2052-6180-1-3 